\title{
Estimating Vaccine Efficacy Against Transmission via Effect on Viral Load
}

\author{
Lee Kennedy-Shaffer, ${ }^{\mathrm{a}}$ Rebecca Kahn, ,,c and Marc Lipsitch ${ }^{\mathrm{b}, \mathrm{c}}$
}

\begin{abstract}
Determining policies to end the SARS-CoV-2 pandemic will require an understanding of the efficacy and effectiveness (hereafter, efficacy) of vaccines. Beyond the efficacy against severe disease and symptomatic and asymptomatic infection, understanding vaccine efficacy against virus transmission, including efficacy against transmission of different viral variants, will help model epidemic trajectory and determine appropriate control measures. Recent studies have proposed using random virologic testing in individual randomized controlled trials to improve estimation of vaccine efficacy against infection. We propose to further use the viral load measures from these tests to estimate efficacy against transmission. This estimation requires a model of the relationship between viral load and transmissibility and assumptions about the vaccine effect on transmission and the progress of the epidemic. We describe these key assumptions, potential violations of them, and solutions that can be implemented to mitigate these violations. Assessing these assumptions and implementing this random sampling, with viral load measures, will enable better estimation of the crucial measure of vaccine efficacy against transmission.
\end{abstract}

Keywords: Coronavirus disease 2019, SARS-CoV-2, Study design, Transmission, Vaccine efficacy

(Epidemiology 2021;32: 820-828)

Submitted May 3, 2021; accepted August 23, 2021

From the apepartment of Mathematics and Statistics, Vassar College, Poughkeepsie, NY; ${ }^{\mathrm{b} C e n t e r}$ for Communicable Disease Dynamics, Harvard T H Chan School of Public Health, Boston, MA; and 'Department of Epidemiology, Harvard T H Chan School of Public Health, Boston, MA.

This work was supported by the Department of Health and Social Care using UK Aid funding and is managed by the NIHR. This work was also supported by the US National Cancer Institute Seronet cooperative agreement U01CA261277. The content is solely the responsibility of the authors and does not necessarily represent the official views of the Department of Health and Social Care or the National Institutes of Health.

L.K.-S. reports no competing interests. M.L. reports consulting/honoraria from Bristol Myers Squibb, Sanofi Pasteur, Janssen, and Merck, as well as a grant through his institution, unrelated to COVID-19, from Pfizer. He has served as an unpaid advisor related to COVID-19 to Pfizer, One Day Sooner, Astra-Zeneca, Janssen, and COVAX (United Biomedical). R.K. discloses consulting fees from Partners in Health.

Correspondence: Lee Kennedy-Shaffer, Department of Mathematics and Statistics, Vassar College, PO Box 226, 124 Raymond Ave, Poughkeepsie, NY 12603. E-mail: LKennedyshaffer@vassar.edu.

Copyright (C) 2021 The Author(s). Published by Wolters Kluwer Health, Inc This is an open access article distributed under the Creative Commons Attribution License 4.0 (CCBY), which permits unrestricted use, distribution, and reproduction in any medium, provided the original work is properly cited.

ISSN: $1044-3983 / 21 / 326-820$

DOI: $10.1097 /$ EDE.0000000000001415
$T^{-}$ understand how vaccinations will affect the severe acute respiratory syndrome coronavirus 2 (SARSCoV-2) pandemic, we must be able to assess both the direct protection and indirect protection that these vaccines confer. ${ }^{1}$ Individually randomized controlled trials (RCTs) have shown high-vaccine efficacy of several vaccine candidates in preventing symptomatic, laboratory-confirmed disease..$^{2-5}$ Several studies have also demonstrated that the vaccines further protect against asymptomatic infection, as measured through virologic or serologic testing of trial participants $\mathrm{s}^{3-5}$ and through repeated sampling in longitudinal cohorts. ${ }^{6}$ However, in the absence of perfect efficacy against acquisition of the infection, fully assessing the effect of vaccination requires an assessment of the vaccine's efficacy at preventing transmission of the pathogen from infected vaccinated individuals to susceptible individuals. ${ }^{7}$ This is especially important for variants, some of which show increased transmissibility, ${ }^{8}$ and in the presence of potential reduced vaccine efficacy against disease for some variants ${ }^{8,9}$ or waning effectiveness with time. ${ }^{10}$

So far, this has been assessed primarily through retrospective observational studies, which have found a reduced risk of infection among household contacts of vaccinated individuals compared with household contacts of unvaccinated individuals. ${ }^{11,12}$ Throughout this article, we discuss estimands that could be estimated in studies that are randomized or observational, "idealized" or "real world." For brevity, we use the term efficacy throughout, to avoid the various distinctions often made between efficacy and effectiveness. ${ }^{13-15}$

Halloran et al. defined vaccine efficacy on infectiousness and its role in understanding the total and overall vaccine effects. ${ }^{7}$ However, methods to estimate this parameter generally focus on large-scale observational studies with observation of contacts of infected individuals, ${ }^{13}$ add-on household studies, genetic linkage studies, or cluster randomized trials. ${ }^{1}$ Rinta-Kokko et al. described methods to estimate vaccine efficacy on carriage prevalence and infer the efficacy on incidence through measured odds ratios. ${ }^{16}$ More recently, similar methods have been proposed to estimate the vaccine efficacy on incident infection, accounting for vaccine effects that change the duration of infection (and thus, likely, the duration of infectiousness) by incorporating random sampling and testing among RCT participants. ${ }^{17,18}$ Follmann and Fay moreover describe how, by measuring 
viral loads or a proxy thereof, the vaccine efficacy on transmission could be estimated. ${ }^{18}$

We describe a method, similar to that laid out in Follmann and Fay, ${ }^{18}$ to estimate vaccine efficacy against transmission by estimating both vaccine efficacy against detectable infection and vaccine efficacy against per-contact infectiousness. This process relies on sampling and virologic testing of the full study population or a random sample thereof within a RCT of a vaccine candidate, as was done in at least one vaccine study for COVID-19. ${ }^{3}$ Additionally, observational studies have used differences in viral load, as measured by the cycle threshold $(\mathrm{Ct})$ value of reverse transcriptase-quantitative polymerase chain reaction (RT-qPCR) tests, to suggest that vaccines may reduce the likelihood of transmission, ${ }^{9,19}$ although perhaps not for the B.1.617.2 (Delta) variant. ${ }^{8,20}$ Although Follmann and Fay focus on the inferential method and statistical properties of such an estimator, ${ }^{18}$ we focus here on assumptions about the infection and transmission process and the vaccine effect that are necessary for consistent estimation of this effect and for proper interpretation of observed differences (or lack thereof) in viral load.

In the next section, we describe the formulation of the estimand of interest and its relation to other common vaccine efficacy measures. In the following section, we define an estimator of this effect and show that it is consistent for that estimand. In the "Assumptions for Consistency" section, we detail the assumptions needed for that consistency, describing their implications, examples of possible violations, and approaches that may be used to mitigate these violations. Finally, we describe the implications of this method and the research needed to best understand when these assumptions are met for SARS-CoV-2 and other infectious pathogens.

\section{ESTIMAND OF INTEREST}

We are interested in the vaccine efficacy in preventing transmission, $V E_{T}$, the reduction in the probability of transmission from an individual at any given time caused by vaccination of that individual. This probability, at any time $t$, can be decomposed as follows:

\section{$P($ transmit at timet $)$ \\ $=P($ infected prior to $t) \times P($ infectious at $t \mid$ infected prior to $t)$ \\ $\times P($ contact susceptible individual at $\mid$ infectious at $)$ \\ $\times P($ transmit at time $t \mid$ infectious and contact susceptible at $t),(1)$}

and so:

$$
V E_{T}=1-\frac{P(\text { transmit at } t \mid \text { vaccinated priortot })}{P(\text { transmit at } t \mid \text { unvaccinated })}
$$

Under the assumptions that: the incidence rate in each arm of the trial is constant across time (Assumption 1); and vaccination status does not affect the probability of having contact with a susceptible individual given that an individual is infectious (Assumption 2), the vaccine efficacy against transmission is given by:

$$
V E_{T}=1-\frac{\lambda_{v} D_{v} \beta_{v}}{\lambda_{u} D_{u} \beta_{u}}
$$

where $\lambda_{v}$ is the incidence rate of infection among vaccinated individuals, $D_{v}$ is the average duration of infectiousness for infected vaccinated individuals, and $\beta_{v}$ is the average percontact transmission probability from an infected vaccinated individual; these quantities with subscript $u$ refer to the quantities among unvaccinated individuals.

Mathematically, this can be rewritten as:

$$
V E_{T}=1-\left(\frac{\lambda_{v}}{\lambda_{u}}\right)\left(\frac{D_{v}}{D_{u}}\right)\left(\frac{\beta_{v}}{\beta_{u}}\right)=1-\left(1-V E_{S}\right)\left(1-V E_{D}\right)\left(1-V E_{\beta}\right),
$$

where $V E_{S}=1-\frac{\lambda_{v}}{\lambda_{u}}$ is the vaccine efficacy against susceptibility or viral acquisition, $V E_{D}=1-\frac{D_{v}}{D_{u}}$ is the vaccine efficacy against duration of infectiousness, and $V E_{\beta}=1-\frac{\beta_{v}}{\beta_{u}}$ is the vaccine efficacy against per-contact infectiousness,

defined as the reduction in the probability of transmission per contact. ${ }^{7,13}$ Halloran et al. use $V E_{I, p}$ where we use $V E_{\beta}$; we avoid that notation to avoid confusion with vaccine efficacy against progression. ${ }^{7,13}$

Under the assumption that an individual is infectious only if they have detectable viral load (Assumption 3), then we can write:

$$
V E_{T}=1-\left(1-V E_{V}\right)\left(1-V E_{\beta}\right),
$$

where $V E_{V}=1-\left(1-V E_{S}\right)\left(1-V E_{D}\right)$ is the vaccine efficacy against prevalent detectable infection, per Lipsitch and Kahn, ${ }^{17}$ and $V E_{\beta}$ is again the vaccine efficacy against percontact infectiousness.

\section{ESTIMATOR USING RANDOM SAMPLES FROM VACCINE RCT}

By equation 5 , to estimate $V E_{T}$, it suffices to estimate $V E_{V}$ and $V E_{\beta}$. In a vaccine RCT, $V E_{V}$ can be estimated using the odds ratio of prevalent detectable infection at time $t$ among vaccinated individuals vs. unvaccinated individuals. ${ }^{16-18}$

If a random sample of participants have virologic tests conducted at some time $t$ after enrollment in an RCT, then the measured viral loads from these test results can be used to estimate the distribution of true viral loads among the vaccinated and the unvaccinated individuals. Note that a nonrandom sample - e.g., tests conducted upon onset of symptoms or upon contact tracing identification, as in Brown et al. ${ }^{8}$-would not provide exactly the distribution of viral loads across the 
course of infection necessary for this approach. ${ }^{21}$ As above, we assume that Assumptions 1-3 hold. If we also assume that the per-contact infectiousness of an individual among vaccinated individuals is some known function $g_{v}(y)$ of the measured viral load $y$, and the per-contact infectiousness of an individual among unvaccinated individuals is some known function $g_{u}(y)$ (Assumption 4), then:

$$
V E_{\beta}=1-\frac{E\left[g_{v}(Y) \mid X=1\right]}{E\left[g_{u}(Y) \mid X=0\right]}
$$

where $Y$ is the measured viral load of a randomly chosen infectious individual and $X$ is the vaccination status of that individual, where $X=1$ denotes a vaccinated individual and $X=0$ an unvaccinated individual. Note that if vaccination only affects infectiousness through its effect on viral load (that is, viral load fully mediates the vaccine efficacy on infectiousness), then $g_{v}=g_{u}$. These assumptions are described in more detail below.

Define the estimator:

$$
\widehat{V E}_{\beta} \equiv 1-\frac{\overline{g_{v}\left(y_{v}\right)}}{\overline{g_{u}\left(y_{u}\right)}},
$$

where $y_{v}$ is the measured viral load of an individual in the vaccinated arm of the trial and $y_{u}$ the measured viral load of an individual in the unvaccinated arm of the trial, and the overbars represent averages across all sampled individuals in the respective arms of the trial. Then if the infectiousness functions $\left(g_{v}\right.$ and $\left.g_{u}\right)$ are correctly specified with respect to the measured viral load in the sample, $\widehat{V E}_{\beta}$ is a consistent estimator of $V E_{\beta}$.

If a measured covariate (e.g., symptom status) modifies the effect of viral load on per-contact infectiousness, this can be incorporated by specifying infectiousness functions for each level of the covariate. For example, let $S$ denote the symptom status of an individual (equal to 1 if symptomatic and 0 if not). Then, if the infectiousness function among symptomatic individuals, whether vaccinated or not, is given by $g_{I}$ and the infectiousness function among individuals not currently symptomatic is given by $g_{0}$, then:

$$
\begin{aligned}
& V E_{\beta}=1-\frac{E\left[g_{S}(Y) \mid X=1\right]}{E\left[g_{S}(Y) \mid X=0\right]}=1 \\
& -\frac{E\left[g_{1}(Y) \mid X=1\right] E[S \mid X=1]+E\left[g_{0}(Y) \mid X=1\right](1-E[S \mid X=1])}{E\left[g_{1}(Y) \mid X=0\right] E[S \mid X=0]+E\left[g_{0}(Y) \mid X=0\right](1-E[S \mid X=0])}
\end{aligned}
$$

This can be estimated by:

$$
\widehat{V E}_{\beta}=1-\frac{\overline{g_{1}\left(y_{v, 1}\right)} \hat{p}_{v, 1}+\overline{g_{0}\left(y_{v, 0}\right)} \hat{p}_{v, 0}}{\overline{g_{1}\left(y_{u, 1}\right)} \hat{p}_{u, 1}+\overline{g_{0}\left(y_{u, 0}\right)} \hat{p}_{u, 0}},
$$

where $y_{i, s}$ represents the measured viral load of an individual in $\operatorname{arm} i$ with symptom status $s$, the overbars represent averages over the sampled individuals in that arm with that symptom status, and $\hat{p}_{i, s}$ is the proportion of individuals in arm $i$ who have symptom status $s$, for $i \in\{u, v\}$ and $s \in\{0,1\}$.

If a study is conducted with randomly sampled virologic tests conducted at a time point $t$, the conditions for a consistent estimator $\widehat{V E}_{V}$ are met, ${ }^{15}$ and the conditions described above for a consistent estimator $\widehat{V E}_{\beta}$ are met, then these can be combined to get a consistent estimator of $V E_{T}$ :

$$
\widehat{V E}_{T}=1-\left(1-\widehat{V E}_{V}\right)\left(1-\widehat{V E}_{\beta}\right)
$$

\section{ASSUMPTIONS FOR CONSISTENCY}

As derived in the previous section, the consistency of $\widehat{V E}_{T}$ as an estimator of $V E_{T}$ relies on four key assumptions. In this section, we describe these assumptions, the consequences of violations of the assumptions, examples of potential violations, and solutions to address these violations. The Table provides a summary of these assumptions.

\section{Assumption 1: Within Each Arm of the Trial, the Incidence Rate Among Participants Is Constant Throughout Time}

Under this assumption, there is a baseline incidence rate within each arm of the vaccine trial, which differs between the arms only due to the vaccine efficacy against susceptibility. If this assumption is violated, then the distribution of viral loads in each arm, $Y_{v}$ and $Y_{u}$, measured by cross-sectional testing at a single point in time are not representative of the distribution of viral loads across the course of infection. ${ }^{21}$

This assumption is violated if the epidemic is growing or waning in the communities in which the trial is conducted. For example, in a growing epidemic, the average time since infection for cases ascertained in a cross-sectional sample is lower than in a flat epidemic. ${ }^{21-23}$ If the vaccine efficacy on viral load mostly affects the later stage of infection-for example, by allowing faster viral clearance, as has been suggested for the Delta variant ${ }^{20}-V E_{\beta}$ will be underestimated in this sample. In a waning epidemic, more individuals will be in the later stage of infection, and so $V E_{\beta}$ will be overestimated in this sample. If, on the other hand, the vaccine efficacy on viral load mostly affects the earlier stage of infection (e.g., by reducing the peak viral load), the reverse will happen: $V E_{\beta}$ will be underestimated in a growing epidemic and overestimated in a waning epidemic. Data on epidemic trajectory could be used to adjust for this bias. Follmann and Fay consider this bias for some viral load functions and vaccine efficacy parameters and find that the bias is less than $10 \%$ for the scenarios considered. ${ }^{18}$

Without adjusting for this bias, the estimate represents the instantaneous vaccine effectiveness on transmission at the time of sampling. That is, the estimand is a function of the epidemic conditions in the community where the trial was conducted. This quantity might be useful in generalizability to communities at a similar stage of their epidemic trajectory. 
This assumption is complicated if there are multiple variants of the pathogen with different incidence rates in the study population. In particular, even if the overall incidence of infection is constant, the assumptions can be violated if one variant is increasing while another is decreasing. If the vaccine has different efficacy levels against the variants, as has been suggested for several SARS-CoV-2 vaccines, ${ }^{8,24-26}$ the observed vaccine efficacy represents a weighted average of these efficacies. However, if the variants have different viral load patterns in infection, as has been suggested for multiple SARS-CoV-2 variants, ${ }^{20,27-29}$ the observed efficacy would then be a weighted average of the specific point-in-time efficacies against each variant at its current growth or decline rate of incidence, which is unlikely to be generalizable to any other population.

\section{Assumption 2: Vaccination Status Does Not Affect the Probability of Contact With a Susceptible Individual at Any Point in Time}

Under this assumption, individuals in both arms have the same distribution of contact rates. If this assumption is violated, then the probability of transmission is no longer proportional to the product of the probability of infection, the mean duration of infectiousness, and the mean per-contact infectiousness (i.e., equations 3 and 4 no longer hold). Correcting for violations of this assumption is challenging as the effects depend on changes in behavior as a result of vaccination.

For example, this violation may occur, even in an RCT with concealed allocation, if vaccination reduces the probability of symptoms given infection (vaccine efficacy for progression to symptoms $>0$ ) and symptomatic individuals are less likely to contact others than asymptomatic individuals. In this case, data on symptom status of cases could be used to adjust for this effect. Additionally, risk compensation of vaccinated individuals - either during nonrandomized vaccine rollout, in an open-label trial, or in a trial with concealed allocation where self-unblinding occurred intentionally ${ }^{30}$ or through strong reactogenicity in the vaccine $\mathrm{arm}^{2,4}$-may affect behavior and contact patterns, violating this assumption. ${ }^{31}$

This assumption is especially important and challenging to ensure in an observational study. During vaccine rollout, vaccinated individuals may have different baseline behaviors from those who are not vaccinated, biasing observational estimates of vaccine effectiveness. Vaccinated individuals may be more likely to interact with other vaccinated individuals, either intentionally or by assortative mixing due to the nature of social networks (e.g., in a cluster randomized trial or during geographically phased vaccination rollout), as has been shown for other vaccines, ${ }^{32}$ or they may engage in risk compensation and relax other precautionary measures. ${ }^{31}$ Contact surveys or proximity data from mobile phones could be used to understand the magnitude of this effect, ${ }^{33,34}$ or it could be estimated by the risk of infection after vaccination but before its effects occur. ${ }^{35}$ These quantitative estimates can then be incorporated into the vaccine efficacy estimate as a component of the probability of contacting a susceptible individual described earlier. These potential changes in behavior and contacts may differentiate the vaccine efficacy measured in an individual RCT from the vaccine effectiveness during a wide rollout or cluster randomized trial; the validity of observational measures of this quantity should thus be considered with caution.

This assumption can be slightly relaxed if the infectiousness function relating measured viral load and per-contact infectiousness actually measures infectiousness accounting for changes in contact patterns that are fully mediated by changes in measured viral load. For example, suppose that higher viral loads are associated with more severe illness, limiting contacts per time infected. If the function $g$ was estimated from a study that estimated secondary attack rate as a function of viral load, ${ }^{36}$ this change in contact pattern, mediated by the viral load, would be incorporated into $g$ and thus into the vaccine efficacy estimate here. Interpretation of $\widehat{V E}_{V}$ should consider the appropriate interpretation of $g$. Figure 1 shows directed acyclic graphs for both versions of this assumption.

\section{Assumption 3: Individuals Without Detectable Viral Load Are Not Infectious}

This assumption implies a perfectly sensitive test for infectiousness. Under a violation of this assumption, for example because of low sensitivity, $\widehat{V E}_{V}$ will be biased. ${ }^{17}$ Adjustments for test characteristics could alleviate the inconsistency in $\widehat{V E}_{V}$. However, the estimate of $\widehat{V E}_{\beta}$ must also be adjusted to account for the infectiousness of undetected infections. This adjustment is less straightforward, as it requires an estimate for the infectiousness of individuals who are infectious but have an undetectable viral load. Sensitivity analysis could be conducted with different assumptions about the infectiousness of these individuals, for example, by assuming they are no more infectious than the average individual with a detectable viral load.

Note that this does not make any assumption about the specificity of the test used. Imperfect specificity can cause bias in $\widehat{V E}_{V}$, but if this is properly accounted for in the infectiousness function, as described under the next assumption, the estimator $\widehat{V E}_{T}$ will remain consistent.

\section{Assumption 4: Vaccination Affects Per-contact Infectiousness Only Through the Measured Viral Load (and Other Measured Covariates); This Relationship Follows a Specified Function or Functions}

Correct specification of the relationship between the measured viral load and infectiousness is crucial to the estimation of $V E_{\beta}$. In other words, the measured viral load acts as a surrogate endpoint for transmission. ${ }^{37}$ Although viral load has been suggested as a proxy for infectiousness of SARSCoV-2, ${ }^{8,36,38-41}$ direct evidence that it meets the formal operational criteria for a surrogate endpoint is limited. There are several reasons this assumption may be violated. 
A

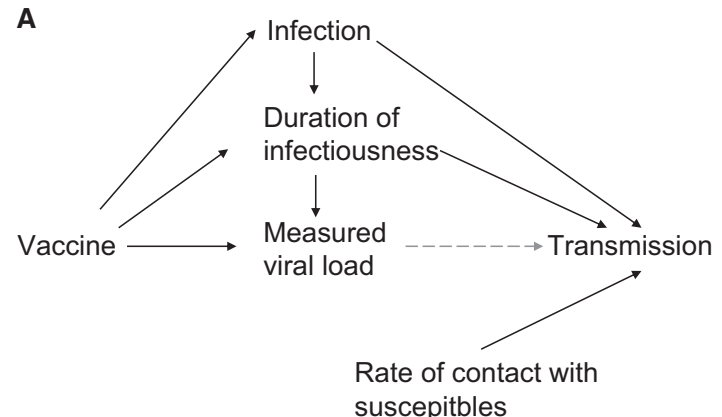

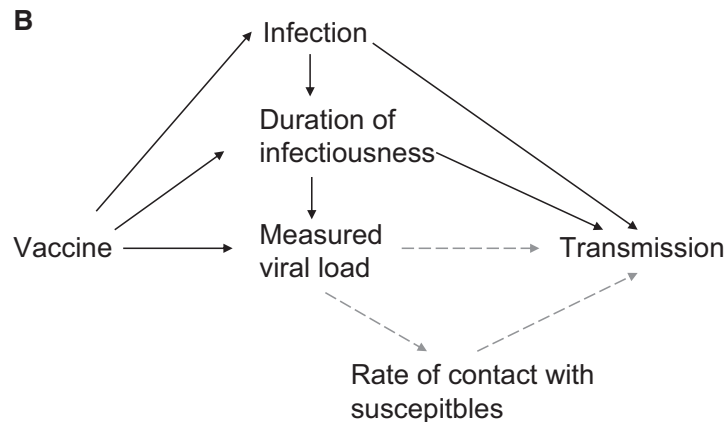

FIGURE 1. Directed acyclic graph for assumption 2. Vaccination can affect transmission through its effect on infection, its effect on duration of infectiousness, and its effect on the measured viral load, a proxy for its effect on infectiousness at any point in time through the infectiousness function $g$ (dashed arrow). The rate of contact with susceptibles, however, is not affected by the vaccine (A). If the rate of contact with susceptibles is affected by the viral load of the infected individual (e.g., through changing contact patterns because of symptoms), the infectiousness function $g$ (dashed arrows) must account for this relationship between measured viral load and transmission probability (B).

First, this assumption would be violated if vaccination reduces the likelihood of being symptomatic at each viral load level and symptomatic patients have higher infectiousness per contact. Data on symptom status could be used to adjust for this and perhaps separate models of the relationship could be used based on symptom status, as described above. This requires, however, the specification of more infectiousness functions.

Second, this assumption would be violated if measured viral load is not an accurate summary of the true viral load and the infectiousness function does not capture this measurement error. This could occur, for example, if the relationship between viral load and infectiousness is estimated using a specific testing platform and the viral loads are measured with a different platform that has different measurement error properties. It could also occur if the relationship is estimated from viral load measurements at a single time point during infection (e.g., symptom onset), as that will be an imperfect representation of the full trajectory of viral loads during infection. As an example, studies culturing viral samples have come to different conclusions about which threshold of $\mathrm{Ct}$ value corresponds with infectious virus, possibly due to different sampling methods, testing platforms, or populations. ${ }^{42,43}$ Ideally, investigators will validate the infectiousness relationship using the same laboratory conditions as in the trial itself.

More generally, the relationship between infectiousness and viral load may be misspecified or unknown, especially for vaccinated individuals. This could occur because the relationship is specified on the wrong scale (e.g., proportional to the $\log$ viral load or $\mathrm{Ct}$ value rather than to the linear viral load, or a threshold effect), because of measurement error (e.g., the discrete nature of $\mathrm{Ct}$ values), or uncertainty in the relationship. It might still be possible, however, to get a range of reasonable estimates for $V E_{\beta}$ by conducting sensitivity analyses with a variety of potential infectiousness functions. $\widehat{V E}_{T}$ can then be bounded by calculating its value with extreme possibilities of these $\widehat{V E}_{\beta}$ values. For example, infectiousness proportional to the viral load and infectiousness constant above a relatively low viral load threshold may be reasonable bounds for most pathogens. These bounds would, for example, encompass a true infectiousness function that is proportional to the log of the viral load.

Violations can occur specifically if vaccination itself affects the relationship between viral load and infectiousness in a way that is not mediated by symptom status or other measured covariates. Often, the only available data to estimate the infectiousness function will be from studies conducted before vaccination, so there may be no ability to account for this difference. For example, Regev-Yochay et al. find that the PCRpositive individuals with a $\mathrm{Ct}$ value below 30 were less likely to test positive for antigen if vaccinated than if unvaccinated. ${ }^{38}$ If, as has been suggested by viral culture studies, ${ }^{44}$ antigen tests are a more accurate reflection of infectiousness than PCR positivity with $\mathrm{Ct}$ values below 30, then this would indicate that vaccines can reduce transmissibility through a mechanism not mediated fully by PCR viral load. If, for any viral load level, a vaccinated individual is no more infectious than an unvaccinated individual, assuming $g_{u}=g_{v}$ would yield a lower bound for $V E_{\beta}$.

Figure 2 shows a directed acyclic graph displaying these assumptions. In practice, this element of the analysis will rely on epidemiologic studies conducted to estimate this relationship,,$^{36,41,45}$ and laboratory analyses that improve biologic understanding of the transmission process. ${ }^{39,40}$ For SARS-CoV-2, these studies suggest a range of possible infectiousness functions: categorical relationships between $\mathrm{Ct}$ values or viral loads and infectiousness, ${ }^{36,45}$ logistic models,,${ }^{39,41}$ and more complicated relationships. ${ }^{40}$ However, these studies are limited in their application to this method because they generally measure viral load at the time of contact tracing identification or onset of symptoms, rather than at a randomly 


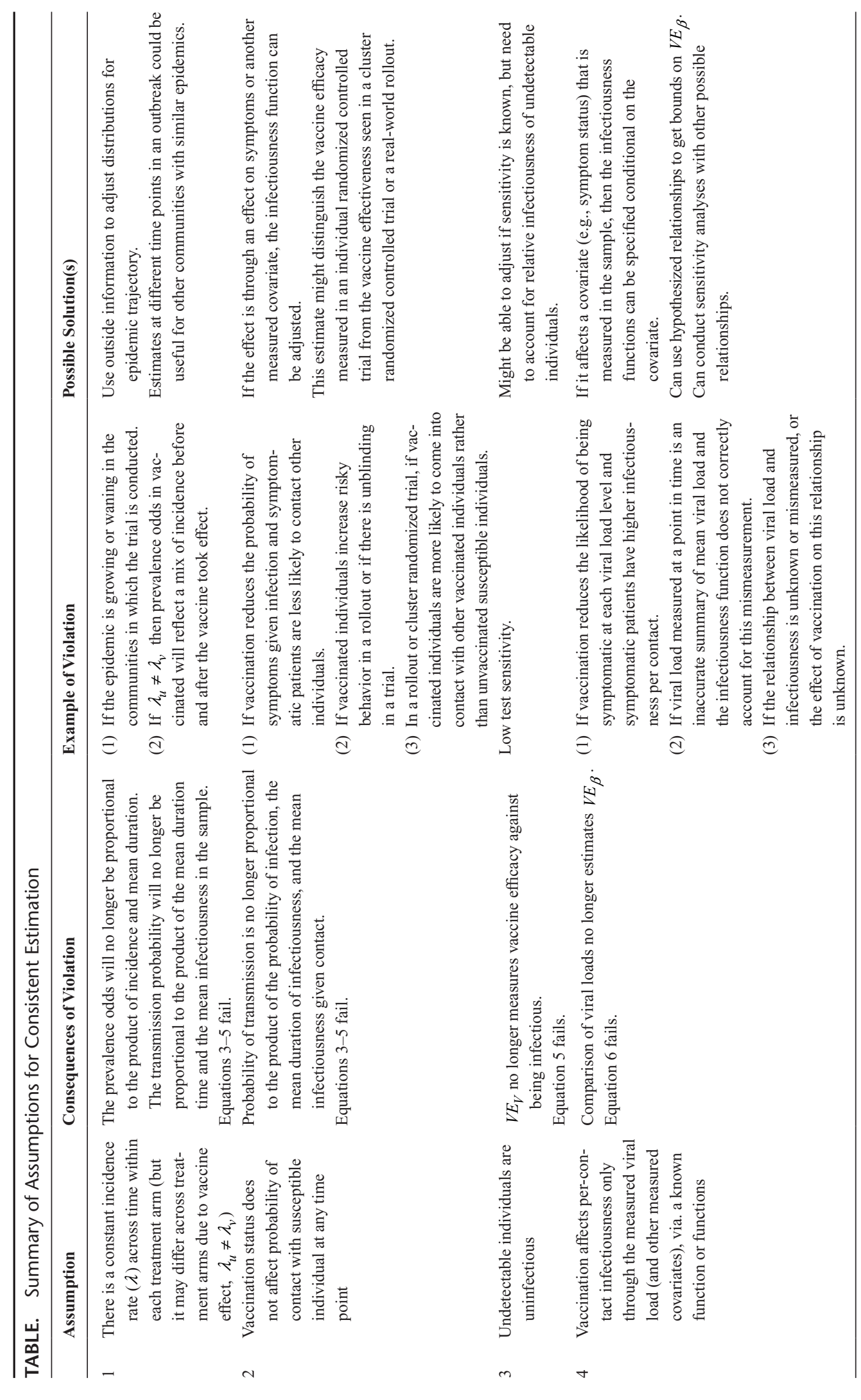




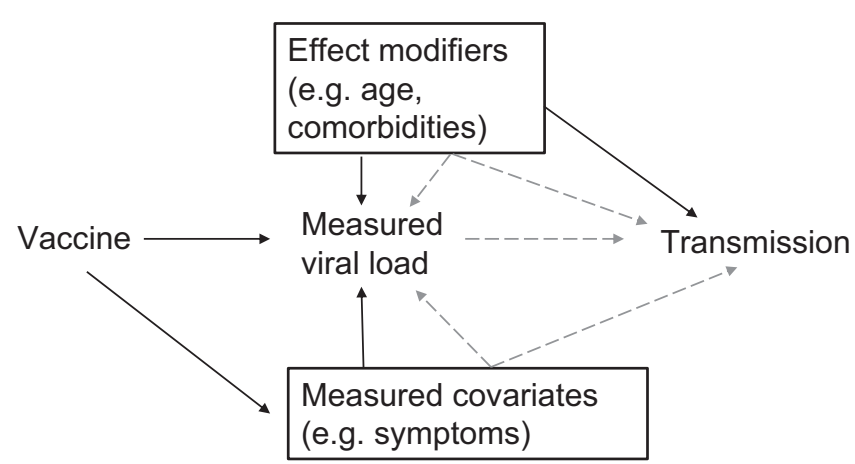

FIGURE 2. Directed acyclic graph for assumption 4. Vaccination can affect transmission only through the measured viral load and measured covariates (e.g., symptom status). Any effect modifiers of the relationship between the measured viral load at any point in time and transmission (e.g., age, comorbidities) are measured and adjusted for through the infectiousness function $g$ (dashed arrows).

sampled time in the duration of infection. Until an epidemiologic study with randomly sampled viral loads is conducted, uncertainty will remain in the measurement of this relationship. Investigators can account for the uncertainty inherent in this relationship via. the sensitivity analyses (e.g., analyzing results using different infectiousness functions) or by formal Bayesian inference that incorporates prior distributions on the infectiousness function or its parameters. How different infectiousness functions will affect the final estimates will depend not only on the structure of those functions but also how the vaccine affects viral loads, and so general bounds on functions are not particularly useful.

\section{DISCUSSION}

Understanding the efficacy of vaccines and how they will affect the trajectory of the SARS-CoV-2 pandemic requires a wide array of studies, both randomized and observational. To get the most information out of large-scale RCTs, these trials should incorporate virologic testing of a random sample of participants, as suggested previously. ${ }^{17,18}$ This allows estimation of vaccine efficacy against prevalent infection, rather than just symptomatic infection. We have shown that, under additional assumptions, using the quantitative information from such random testing additionally allows for the estimation of vaccine efficacy against transmission, a crucial parameter for understanding how widespread vaccination will affect circulating virus.

This estimation requires four key assumptions. First, the incidence rate among participants must be constant throughout time within each arm of the trial. Second, vaccination status must not affect the probability of contact with a susceptible individual during the study. Third, individuals without a detectable viral load are assumed to be uninfectious. And fourth, vaccination must affect infectiousness only through a known function of the measured viral load and, potentially, other measured covariates.
Meeting the assumptions for this estimation requires an understanding of the role of viral load in the transmission process. This increased understanding of viral load and proxies such as the $\mathrm{Ct}$ value measured by RT-qPCR tests can contribute to better understanding of the pandemic in several ways, including monitoring population incidence, ${ }^{21}$ assessing the role of variants in outbreaks, ${ }^{8,27-29}$ assessing test performance, ${ }^{46}$ and clinical management. ${ }^{47}$ Although some studies have assessed the association between measured viral load and infectiousness, ${ }^{36,41,45}$ more such work is needed to improve this aspect of the estimation.

For SARS-CoV-2, existing studies can be used to assess these assumptions, but in any specific setting they are likely unprovable. For assumption 1, running the trial during a time period where incidence is flat is key; if this is not possible, investigators should acknowledge the lack of generalizability of the observed $\mathrm{Ct}$ values ${ }^{21}$ and the uncertainty that brings, as shown elsewhere. ${ }^{18}$ In the closed-label individually randomized trial setting, assumption 2 is similar to a general assumption of exchangeability in RCTs. The generalizability to a wide vaccine rollout, however, depends on behavioral patterns and an assumption that risk compensation will not occur. Assumption 3 relies on the sensitivity of the test, which has been well-established for SARS-CoV-2. ${ }^{46}$ Assumption 4, the most challenging assumption, can be estimated from a variety of existing studies, ${ }^{36,39-41,45}$ which can be used to provide a range of estimates for the vaccine efficacy. If feasible, a parallel study to the main trial specifically designed to determine the infectiousness function for the test instrument used in the trial on the viral population circulating at that time, for example, by culturing or epidemiologic tracing, would be beneficial and allow more precise specification by investigators.

This method could be used to compare the efficacy of two different vaccines or vaccine regimens and in observational studies, in which control of confounding and avoidance of selection biases will present challenges similar to, but slightly more extensive than, the ones in standard vaccine effectiveness studies. ${ }^{48}$ In addition to the four main assumptions, such an analysis would require exchangeability assumptions: that there is no unmeasured confounding between vaccination status and any of the following: the probability of infection, duration of infection, measured viral load, and the relationship between viral load and infectiousness. Moreover, for a retrospective study, selection criteria would need to not exclude individuals because of any factor-for example, hospitalization or death-causally related to vaccination and measured viral load ${ }^{49}$ and would need random sampling of viral loads across the course of infections.

Limiting the spread of SARS-CoV-2 variants, ending the SARS-CoV-2 pandemic, and mitigating future pandemics will require the best available evidence, as quickly as possible, on the full set of measures of vaccine efficacy. 
We have described a method to gain a richer picture of vaccine efficacy on transmission with the optimal use of quantitative data from virologic testing on a sample of trial participants.

\section{REFERENCES}

1. Lipsitch M, Dean NE. Understanding COVID-19 vaccine efficacy. Science. 2020;370:763-765.

2. Polack FP, Thomas SJ, Kitchin N, et al.; C4591001 Clinical Trial Group. Safety and Efficacy of the BNT162b2 mRNA Covid-19 Vaccine. $N$ Engl J Med. 2020;383:2603-2615.

3. Voysey M, Clemens SAC, Madhi SA, et al.; Oxford COVID Vaccine Trial Group. Safety and efficacy of the ChAdOx1 nCoV-19 vaccine (AZD1222) against SARS-CoV-2: an interim analysis of four randomised controlled trials in Brazil, South Africa, and the UK. Lancet. 2021;397:99-111.

4. Baden LR, El Sahly HM, Essink B, et al.; COVE Study Group. Efficacy and Safety of the mRNA-1273 SARS-CoV-2 Vaccine. $N$ Engl $J$ Med. 2021;384:403-416.

5. Oliver SE, Gargano JW, Scobie H, et al. The advisory committee on immunization practices' interim recommendation for use of Janssen COVID-19 Vaccine - United States, February 2021. MMWR Morb Mortal Wkly Rep. 2021;70:329-332.

6. Thompson MG, Burgess JL, Naleway AL, et al. Interim estimates of vaccine effectiveness of BNT162b2 and mRNA-1273 COVID-19 vaccines in preventing SARS-CoV-2 infection among health care personnel, first responders, and other essential and frontline workers - Eight U.S. Locations, December 2020-March 2021. MMWR Morb Mortal Wkly Rep. 2021;70:495-500.

7. Halloran ME, Longini IM Jr, Struchiner CJ. Design and interpretation of vaccine field studies. Epidemiol Rev. 1999;21:73-88.

8. Brown CM, Vostok J, Johnson H, et al. Outbreak of SARS-CoV-2 infections, including COVID-19 vaccine breakthrough infections, associated with large public Gatherings - Barnstable County, Massachusetts, July 2021. MMWR Morb Mortal Wkly Rep. 2021;70:1059-1062.

9. Pritchard E, Matthews PC, Stoesser N, et al. Impact of vaccination on new SARS-CoV-2 infections in the United Kingdom. Nat Med. 2021;27:1370-1378.

10. Thomas SJ, Moreira ED, Kitchin N, et al. Six month safety and efficacy of the BNT162b2 mRNA COVID-19 vaccine. medRxiv Preprint 2021; doi: 10.1101/2021.07.28.21261159.

11. Shah ASV, Gribben C, Bishop J, et al. Effect of vaccination on transmission of COVID-19: an observational study in healthcare workers and their households. medRxiv Preprint 2021; doi: 10.1101/2021.03.11.21253275.

12. Harris RJ, Hall JA, Zaidi A, Andrews NJ, Dunbar JK, Dabrera G. Effect of vaccination on household transmission of SARS-CoV-2 in England. $N$ Engl J Med. 2021;385:759-760.

13. Halloran ME, Longini IM, Struchiner CJ. Design and Analysis of Vaccine Studies. Springer; 2010.

14. Gartlehner G, Hansen RA, Nissman D, Lohr KN, Carey TS. Criteria for Distinguishing Effectiveness from Efficacy Trials in Systematic Reviews. AHRQ Publication No. 06-0046. Agency for Healthcare Research and Quality; 2006.

15. Singal AG, Higgins PD, Waljee AK. A primer on effectiveness and efficacy trials. Clin Transl Gastroenterol. 2014;5:e45

16. Rinta-Kokko H, Dagan R, Givon-Lavi N, Auranen K. Estimation of vaccine efficacy against acquisition of pneumococcal carriage. Vaccine. 2009;27:3831-3837.

17. Lipsitch M, Kahn R. Interpreting vaccine efficacy trial results for infection and transmission. Vaccine. 2021;39:4082-4088.

18. Follmann D, Fay M. Vaccine efficacy at a point in time. medRxiv Preprint 2021; doi: 10.1101/2021.02.04.21251133.

19. Levine-Tiefenbrun M, Yelin I, Katz R, et al. Initial report of decreased SARS-CoV-2 viral load after inoculation with the BNT162b2 vaccine Nat Med. 2021;27:790-792.

20. Chia PY, Ong SWX, Chiew CJ, et al. Virological and serological kinetics of SARS-CoV-2 Delta variant vaccine-breakthrough infections: a multi-center cohort study. medRxiv Preprint 2021; doi: 10.1101/2021.07.28.21261295.
21. Hay JA, Kennedy-Shaffer L, Kanjilal S, et al. Estimating epidemiologic dynamics from cross-sectional viral load distributions. Science. 2021;373:eabh0635.

22. Wallinga J, Lipsitch M. How generation intervals shape the relationship between growth rates and reproductive numbers. Proc Biol Sci. 2007;274:599-604.

23. Rydevik G, Innocent GT, Marion G, et al. Using combined diagnostic test results to hindcast trends of infection from cross-sectional data. PLoS Comput Biol. 2016;12:e1004901.

24. Madhi SA, Baillie V, Cutland CL, et al.; NGS-SA Group; Wits-VIDA COVID Group. Efficacy of the ChAdOx1 nCoV-19 Covid-19 Vaccine against the B.1.351 Variant. $N$ Engl J Med. 2021;384:1885-1898.

25. Mahase E. Covid-19: Novavax vaccine efficacy is $86 \%$ against UK variant and $60 \%$ against South African variant. BMJ. 2021;372:n296.

26. Planas D, Bruel T, Grzelak L, et al. Sensitivity of infectious SARSCoV-2 B.1.1.7 and B.1.351 variants to neutralizing antibodies. Nat Med. 2021;27:917-924.

27. Kidd M, Richter A, Best A, et al. S-Variant SARS-CoV-2 Lineage B1.1.7 Is associated with significantly higher viral load in samples tested by TaqPath polymerase chain reaction. J Infect Dis. 2021;223:1666-1670.

28. Kissler SM, Fauver JR, Mack C, et al. Densely sampled viral trajectories for SARS-CoV-2 variants alpha (B.1.1.7) and epsilon (B.1.429). medRxiv Preprint 2021; doi: 10.1101/2021.02.16.21251535.

29. Faria NR, Mellan TA, Whittaker C, et al. Genomics and epidemiology of the P.1 SARS-CoV-2 lineage in Manaus, Brazil. Science. 2021;372:815-821.

30. Dotinga R. Self-unblinding threatens COVID vaccine trial. MedPage Today. 2021. Available at: https://www.medpagetoday.com/specialreports/exclusives/91584. Accessed August 3, 2021.

31. Trogen B, Caplan A. Risk compensation and COVID-19 vaccines. Ann Intern Med. 2021;174:858-859.

32. Masters NB, Eisenberg MC, Delamater PL, Kay M, Boulton ML, Zelner J. Fine-scale spatial clustering of measles nonvaccination that increases outbreak potential is obscured by aggregated reporting data. Proc Natl Acad Sci U S A. 2020;117:28506-28514.

33. Andersson O, Campos-Mercade P, Meier A, Wengström E. Anticipation of COVID-19 vaccines reduces social distancing. SSRN Preprint 2021; doi: $10.2139 /$ ssrn.3765329.

34. Nguyen V, Liu Y, Mumford R, et al. Changes in mobility pre and post first SARS-CoV-2 vaccination: findings from a prospective community cohort study including GPS movement tracking in England and Wales (Virus Watch). medRxiv Preprint 2021; doi: 10.1101/2021.06.21.21259237.

35. Hoehl S, Ciesek S, Graf J, Wicker S. A new group at increased risk of a SARS-CoV-2 infection emerges: the recently vaccinated. Vaccine. 2021;39:4025-4026.

36. Lee LYW, Rozmanowski S, Pang M, et al. SARS-CoV-2 infectivity by viral load, $\mathrm{S}$ gene variants and demographic factors and the utility of lateral flow devices to prevent transmission. Clin Infect Dis 2021:ciab421. doi: 10.1093/cid/ciab421.

37. Prentice RL. Surrogate endpoints in clinical trials: definition and operational criteria. Stat Med. 1989;8:431-440.

38. Regev-Yochay G, Amit S, Bergwerk M, et al. Decreased infectivity following BNT162b2 vaccination: a prospective cohort study in Israel. Lancet Reg Health Eur. 2021;7:100150.

39. Singanayagam A, Patel M, Charlett A, et al. Duration of infectiousness and correlation with RT-PCR cycle threshold values in cases of COVID-19, England, January to May 2020. Euro Surveill. 2020;25:2001483.

40. Jones TC, Biele G, Mühlemann B, et al. Estimating infectiousness throughout SARS-CoV-2 infection course. Science. 2021;373:eabi5273.

41. Marks M, Millat-Martinez P, Ouchi D, et al. Transmission of COVID19 in 282 clusters in Catalonia, Spain: a cohort study. Lancet Infect Dis. 2021;21:629-636.

42. Bullard J, Dust K, Funk D, et al. Predicting infectious severe acute respiratory syndrome coronavirus 2 from diagnostic samples. Clin Infect Dis. 2020;71:2663-2666.

43. La Scola B, Le Bideau M, Andreani J, et al. Viral RNA load as determined by cell culture as a management tool for discharge of SARS-CoV-2 patients from infectious disease wards. Eur J Clin Microbiol Infect Dis. 2020;39:1059-1061 
44. Pekosz A, Parvu V, Li M, et al. Antigen-based testing but not real-time polymerase chain reaction correlates with severe acute respiratory syndrome coronavirus 2 viral culture. Clin Infect Dis. 2021:ciaa1706. doi: $10.1093 / \mathrm{cid} / \mathrm{ciaa} 1706$.

45. Mitjà $\mathrm{O}$, Corbacho-Monné $\mathrm{M}$, Ubals M, et al.; BCN-PEP-CoV2 Research Group. A cluster-randomized trial of hydroxychloroquine for prevention of COVID-19. N Engl J Med. 2021;384:417-427.

46. Tom MR, Mina MJ. To interpret the SARS-CoV-2 test, consider the cycle threshold value. Clin Infect Dis. 2020;71:2252-2254.
47. Moraz M, Jacot D, Papadimitriou-Olivgeris M, et al. Universal admission screening strategy for COVID-19 highlighted the clinical importance of reporting SARS-CoV-2 viral loads. New Microbes New Infect. 2020;38:100820.

48. Lipsitch M, Jha A, Simonsen L. Observational studies and the difficult quest for causality: lessons from vaccine effectiveness and impact studies. Int J Epidemiol. 2016;45:2060-2074.

49. Accorsi EK, Qiu X, Rumpler E, et al. How to detect and reduce potential sources of biases in studies of SARS-CoV-2 and COVID-19. Eur J Epidemiol. 2021;36:179-196. 